\title{
Click Chemistry Approach to Structurally Simplified Pancratistatin Analogs
}

\author{
V. de la Sovera, A. Bellomo and D. Gonzalez.
}

Departamento de Química Orgánica, Facultad de Química,UdelaR, Gral. Flores 2124, Montevideo, Uruguay. *vdelasovera@fq.edu.uy

Keywords: biological activity, 1,3-dipolar cycloaddition, biocatalysis.

\section{INTRODUCTION}

The alkaloid pancratistatin isolated from the Polynesian plant Pancratium litoralle exhibits strong antitumor, antiviral, and antiparasitic activity. ${ }^{1}$ The mechanism of action has not been fully elucidated but it is known that the compound acts as a selective apoptosis inducer in tumor cells without affecting the normal tissue. There are several syntheses of pancratistatin but none of the available methods has been adapted to produce large quantities of material which cannot be obtained efficiently from the natural source either. In that sense, research has focused in the synthesis of simplified pancratistatin analogs. ${ }^{2}$ In this opportunity, we present our more recent results in the construction of a tricyclic analog of the Amarillidaceae alkaloids of the pancratistatin and narciclasine group by means of a strategy that involves biocatalysis and Huisgen cycloaddition.

\section{RESULTS AND DISCUSSION}

Our approach starts with an enzymatic step where bromobenzene is converted into the optically pure metabolite 2. This compound is then transformed into azide 4 by a well established methodology (Scheme 1). ${ }^{3}$

At this point the route diverges and two different strategies were explored. Both involved esterification and Huisgen cycloaddition but the order of the events is switched (routes A and B in Scheme 2). We have previously explored and optimized these reactions in the brominated conduritol analogs. ${ }^{4}$ With the tricyclic core 7 in our hands we studied the subsequent ruthenium catalyzed dihydroxylation and deprotection reactions in order to obtain the desired alkaloid analogs.

Scheme 1. Chemoenzymatic synthesis of compound 4.
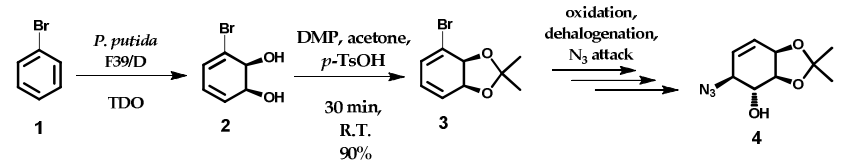

Scheme 2. Designed routes for the synthesis of pancratistatin analogs.

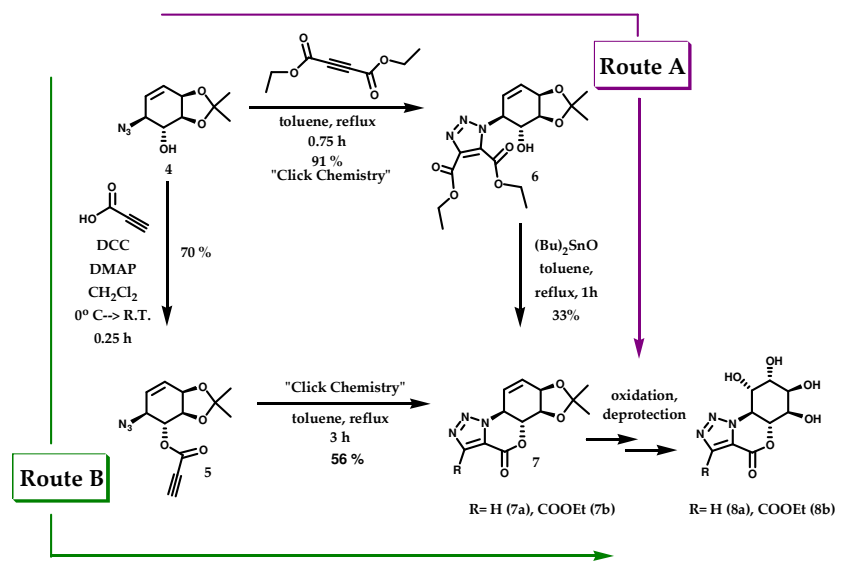

\section{CONCLUSION}

We have successfully prepared two lactonic pancratistatin analogs (7a and $7 \mathbf{b})$. These compounds will be subjected to biological essays to evaluate their activity. In addition they are model structures for the synthesis of the corresponding lactams which more closely resemble the natural alkaloid.

\section{ACKNOWLEDGEMENTS}

Victoria acknowledges PEDECIBA and ANII (FCE252) for the corresponding scholarships.

We are particularly thankful to Horacio Pezzaroglo (NMR spectra), and Jorge Adum for carrying out the fermentation process.

\section{REFERENCES}

${ }^{1}$ Kornienko A.; Evidente A., Chem. Rev. 2008, 108, 1982-2014.

${ }^{2}$ Rinner, U.; Hillebrenner, H. L.; Adams, D. R.; Hudlicky, T.; Pettit, G. R., Bioorganic \& Medicinal Chemistry Letters 2004, 14, 2911 2915.

${ }^{3}$ Bellomo, A.; Gonzalez, D., Tetrahedron: Asymmetry 2006, 17, 474-478.

${ }^{4}$ de la Sovera V.; Bellomo A.; Gonzalez D. Tetrahedron Lett. 2011, 52, 430-433. 